\title{
Kidney Transplantation With Corticosteroid-Free Maintenance Immunosuppression: A Single Center Analysis of Graft and Patient Survivals
}

\author{
R. Filipe, A. Mota, R. Alves, C. Bastos, F. Macário, A. Figueiredo, A. Roseiro, B. Parada, H. Sá,
} P. Nunes, and M. Bastos

\begin{abstract}
The purpose of this study was to assess the impact of a corticosteroid-free maintenance immunosuppression on graft survival in kidney transplantation. We analyzed 79 patients who were transplanted between June 1, 2006 and May 31, 2007. We excluded hyperimmunized patients, second transplantations, living donors, and black recipients. Patients underwent induction with thymoglobulin or basiliximab, followed by treatment with mycophenolate mofetil (MMF), tacrolimus, and methylprednisolone. On the 5th day, the patients were divided into 2 groups: group $\mathrm{A}(\mathrm{n}=45)$ discontinued steroid therapy; group $\mathrm{B}(\mathrm{n}=34)$ continued prednisone therapy. We performed a comparative analysis of incidence of delayed graft function (DGF), acute rejection episodes (ARE), renal function at 6 and 12 months, graft and patient survivals, causes of graft loss, and mortality. The 2 groups were similar for donor, recipient, and graft characteristics. The incidences of DGF were $8.9 \%$ in group A and $14.7 \%$ in group B; those for ARE were $2.3 \%$ in group A and $13.8 \%$ in group $\mathrm{B}(P=.077)$. The mean serum creatinine levels at 6 and 12 months were similar. There were 8 graft losses: 3 in group A ( 3 deaths with functioning grafts) and 5 in group B (1 death, 3 vascular causes, 1 kidney nonfunction). The 4 deaths were due to infection $(\mathrm{n}=3)$ or neoplasia $(\mathrm{n}=1)$. Graft survivals at 1 year were $98 \%$ in group A and $85 \%$ in group B, and patient survivals were $98 \%$ and $97 \%$, respectively. An immunosuppressive regimen using antibody induction and steroid-free treatment proved to be effective in low-risk patients.
\end{abstract}

From the Renal Transplantation Unit, Departments of Urology and Kidney Transplantation (R.F., A.M., C.B., A.F., A.R., B.P., P.N., M.B.) and Nephrology (R.A., F.M., H.S.), Coimbra University Hospital, Coimbra, Portugal.
Address reprint requests to $\mathrm{R}$. Filipe, Coimbra University Hospital, Department of Urology and Kidney Transplantation, Coimbra, Portugal. E-mail: rmafilipe@gmail.com

$0041-1345 / 09 / \$-$ see front matter doi:10.1016/j.transproceed.2009.02.001 
$\mathrm{C}$ ORTICOSTEROIDS are the only immunosuppressants in current use that have been prescribed since the beginning of allotransplantation. Unfortunately, they are responsible for a great deal of morbidity among transplant patients, including increased susceptibility to infection; weight gain with cushingoid changes; cataracts; glucose intolerance; myopathy; sodium retention; hyperlipidemia; osteopenia; aseptic necrosis; and impaired growth. ${ }^{1}$ Additionally, these side effects contribute to noncompliance in some patients.

The appearance of antimetabolites (azathioprine and later mycophenolate mofetil [MMF]) and calcineurin inhibitors (cyclosporine, and more recently tacrolimus) has permitted more specific immunosuppression, with possibilities of corticosteroid-sparing. Besides these improvements, elimination of steroids has not been free of risk. Initial studies with early corticoid withdrawal revealed higher incidences of acute rejection episodes, ${ }^{2-5}$ but the use of antibody induction therapy permitted more potent and specific immunosuppression, ${ }^{6-9}$ allowing greater safety for early corticoid withdrawal.

Immunosuppression schemes with rapid suspension of steroids ( 1 week) show advantages over late suspension: it is easier to diagnose acute rejection episode (ARE), there is less interference in the mechanisms of immune tolerance, there are fewer side effects, and the lack of dependence on steroids prevents the increased immune activity secondary to late steroid withdrawal.

\section{MATERIALS AND METHODS \\ Patients and Study Design}

In this retrospective, single center study, we collected data from 79/104 kidney transplantations performed between June 2006 and May 2007, excluding hyperimmunized (PRA > 75\%) patients, second transplantation, recipients of living donor grafts, and black subjects. Two groups were established: group A $(n=45)$ with steroid withdrawal on day 5 versus group $\mathrm{B}(\mathrm{n}=34)$ with maintenance steroids. Besides steroids, the remaining immunosuppressants and adjuvant therapy were the same.

\section{Immunologic Evaluation}

Cross match and PRA determination were performed by flow cytometry. HLA A, B, and DR mismatches were determined in all recipients.

\section{Immunosuppression}

All patients received induction therapy with a randomly chosen antibody: thymoglobulin (anti-human thymocyte globulin; Genzyme) or basiliximab (anti-CD25 monoclonal antibody; Novartis). Patients received a 5-day course of thymoglobulin $(1.5 \mathrm{mg} / \mathrm{kg})$ with the initial infusion begun just before surgery. The following doses were adjusted to the degree of myelosuppression. Basiliximab was prescribed at a dose of $20 \mathrm{mg}$ before surgery and on postoperative day 4 . No dose adjustments were made. Thymoglobulin was given to $51 \%$ of group A patients and $36 \%$ of group B; the other subjects received basiliximab.

Oral MMF (1 g twice a day) was started before and maintained after surgery. The MMF dose was adjusted for gastrointestinal side effects or bone marrow suppression. Tacrolimus was given to achieve a trough concentration between 8 and $12 \mathrm{ng} / \mathrm{mL}$; the initial dose was $0.15 \mathrm{mg} / \mathrm{kg} / \mathrm{d}$, which was adjusted every other day according to the blood levels. All patients received $500 \mathrm{mg}$ methylprednisolone on the day of transplantation, $250 \mathrm{mg}$ on day 2 , and $125 \mathrm{mg}$ on day 4. On postoperative day 5, group A patients discontinued corticoids, and group B maintained them with a prednisone taper as usual to achieve $30 \mathrm{mg}$ daily at day 7 and $15 \mathrm{mg}$ at day 30 .

\section{Infection Prophylaxis}

Cytomegalovirus (CMV) prophylaxis was administered as valganciclovir (450 mg daily for 6 months) to all patients who received thymoglobulin. Pneumocystis prophylaxis included trimethropimsulfamethoxazole ( $480 \mathrm{mg}$ daily) to all patients for 1 year. All patients were HIV, hepatitis $\mathrm{C}$ and $\mathrm{B}$ negative, and CMV positive.

\section{Data Analysis}

We compared patient and graft survival rates and the incidence and timing of ARE. Graft failure was defined by death with a functioning graft, by a return to dialysis, or by retransplantation. We also compared the incidence of delayed graft function (DGF), donor and recipient characteristics, renal function at 6 and 12 months, causes of graft loss and mortality, HLA mismatches, posttransplant diabetes mellitus, and allograft characteristics. All rejection episodes were diagnosed by biopsy and classified using Banff 97 criteria. Recipient and donor characteristics and incidences of intercurrent events were compared using chi-square tests. For continuous variables we used the standard 2 sample student $t$ test. Actuarial rates for graft and patient survivals were estimated using Kaplan-Meier methods and compared using log-rank tests. Differences were considered significant if $P<.05$.

\section{RESULTS}

\section{Baseline Characteristics}

The mean patient follow-up was $1.48 \pm 0.48$ years, with no difference between groups. The baseline characteristics of the 2 groups were similar. The donors showed similar ages, creatininemia, and weights. Trauma was the principal cause of donor death in both groups (Table 1). The incidence of diabetic recipients was $13 \%$ in group $\mathrm{A}$ and $15 \%$ in group $\mathrm{B} ;>80 \%$ of patients had associated pathology (mainly cardiovascular). Time on dialysis, age, gender, and weight of recipients were similar in both groups.

The allograft characteristics did not differ significantly between the group with corticosteroid maintenance vs withdrawal: cold ischemia times were $17.2 \pm 5.5$ hours in group $\mathrm{A}$ and $17.5 \pm 5.6$ hours in group $\mathrm{B}(P=.83)$, and the mean mismatches were similar. In group $A$ the transplanted kidney was more frequently the left $(56.8 \%)$, and in group B the right kidney $(52.9 \%)$, but without significance $(P=.31)$.

\section{ARE}

In the group with early corticosteroid withdrawal, 1 patient $(2.3 \%)$ developed an ARE (grade IA), and in the group with corticoid maintenance, 4 patients $(13.8 \% ; P=.077)$ experienced ARE (grade IA). All AREs were treated with methylprednisolone pulses. 
Table 1. Baseline Characteristics and Results of Study Groups

\begin{tabular}{lccl}
\hline & Group A & Group B & $P$ \\
\hline Donor & & & \\
Age (y) & $40.44 \pm 17.2$ & $38.68 \pm 17.8$ & .94 \\
Gender (\% males) & 53.3 & 55.8 & .63 \\
Weight (kg) & $69.98 \pm 14.5$ & $68.12 \pm 12.9$ & .37 \\
Cause of death (\% trauma) & 38 & 43 & .16 \\
Creatininemia (mg/dL) & $0.8 \pm 0.2$ & $0.8 \pm 0.2$ & .86 \\
Recipient & & & \\
Age (y) & $47.13 \pm 13.0$ & $46.91 \pm 13.3$ & .94 \\
Gender (\% males) & 64 & 61 & .8 \\
Weight (kg) & $65.58 \pm 11.8$ & $63.12 \pm 12.4$ & .37 \\
Associated pathology (\%) & 91.1 & 82.3 & .31 \\
$\quad$ Diabetes mellitus (\%) & 13 & 15 & .8 \\
Time on dialysis (mo) & $44.76 \pm 29.5$ & $56.82 \pm 52.4$ & .20 \\
Cold ischemia time (h) & $17.2 \pm 5.5$ & $17.5 \pm 5.6$ & .83 \\
HLA mismatches & $2.54 \pm 0.90$ & $2.50 \pm 1.2$ & .88 \\
Transplanted kidney & $56.8 / 43.2$ & $47.1 / 52.9$ & .31 \\
$\quad$ (\% left/\% right) & & & \\
Delayed graft function (\%) & 8.9 & 14.7 & .48 \\
Acute rejection episodes (\%) & 2.3 & 13.8 & .077 \\
Creatininemia at 6 months & $1.29 \pm 0.29$ & $1.28 \pm 0.35$ & .91 \\
$\quad$ (mg/dL) & & & \\
Creatininemia at 12 months & $1.30 \pm 0.48$ & $1.32 \pm 0.49$ & .84 \\
$\quad$ (mg/dL) & & & \\
Graft survival at 1 year (\%) & 98 & 85 & .20 \\
Patient survival at 1 year (\%) & 98 & 97 & .57 \\
Post transplant diabetes & 18 & 17 & .51 \\
$\quad$ mellitus (\%) & & & \\
\hline & & & \\
\hline
\end{tabular}

\section{Graft Function}

Both groups showed similar incidences of DGF: $8.9 \%$ in group $\mathrm{A}$ and $14.7 \%$ in group $\mathrm{B}(P=.48)$. The renal function was similar: group A: $1.29 \pm 0.29 \mathrm{mg} / \mathrm{dL}$ versus group B: $1.28 \pm 0.36 \mathrm{mg} / \mathrm{dL}(P=.91)$ at 6 months and group A: $1.30 \pm 0.48 \mathrm{mg} / \mathrm{dL}$ versus group $\mathrm{B}: 1.32 \pm 0.49$ $\mathrm{mg} / \mathrm{dL}(P=.84)$ at 12 months.

\section{Posttransplant Diabetes Mellitus}

There were no differences between the groups concerning the incidences of posttransplant diabetes mellitus: $18 \%$ in group $\mathrm{A}$ and $17 \%$ in group $\mathrm{B}$.

\section{Patient and Graft Survivals}

There were 8 graft losses: 3 in group A (all deaths with functioning grafts) and 5 in group B (1 death, 3 vascular causes, 1 kidney nonfunction). The 4 deaths ( 2 in the first year and 2 in the second year) were due to septic shock $(\mathrm{n}=$ $2)$, pneumonia $(n=1)$ and cervical tumor $(n=1)$.

The 12-month outcomes showed no significant differences: 12-month graft survivals of group A versus group B were $98 \%$ vs $85 \%(\log$-rank $P=.20)$, and 12 -month patient survivals were $98 \%$ versus $97 \%$ (log-rank $P=.57$ ), respectively.

\section{DISCUSSION}

Our study was designed to demonstrate the efficacy of an immunosuppressive regimen with antibody induction and steroid-free immunosuppression in low-risk patients. We observed good acute rejection rates, initial graft function, as well as graft and patient survivals. The features of the 2 groups, 1 with corticoid maintenance and another with corticoid withdrawal at day 5 , showed similar characteristics. The main difference was the greater incidence of ARE among patients with corticoid maintenance, albeit not significant. We also have to point out that thymoglobulin was the major treatment in the corticosteroid withdrawal group, albeit not significant. With respect to the incidence of corticosteroid side effects, their avoidance did not improve the incidence of posttransplant diabetes mellitus.

In conclusion, low-risk patients are adequately treated with immunosuppressive regimens including antibody induction and early discontinuation of steroids, with good ARE rates, as well as graft and patient survivals. Longer follow-up of these groups is required to evaluate the advantages and chronic rejection incidence associated with a steroid-free maintenance scheme. ${ }^{10}$

\section{REFERENCES}

1. Augustine JJ, Hricik DE: Steroid sparing in kidney transplantation: hanging paradigms, improving outcomes, and remaining questions. Clin J Am Soc Nephrol 1:1080, 2006

2. Hricik DE, O'Toole M, Schulak JA, et al: Steroid-free immunosuppression in cyclosporine-treated renal transplant recipients: a meta-analysis. J Am Soc Nephrol 4:1300, 1993

3. Schulak JA, Mayes JT, Moritz CE, et al: A prospective randomized trial of prednisone versus no prednisone maintenance therapy in cyclosporine and azathioprine treated renal transplant patients. Transplantation 49:327, 1990

4. Hricik DE, Whalen C, Lautman J, et al: Withdrawal of steroids after renal transplantation: clinical predictors of outcome. Transplantation 53:41, 1992

5. Stratta RJ, Armbrust MJ, Oh CS, et al: Withdrawal of steroid immunosuppression in renal transplant recipients. Transplantation 45:323, 1988

6. Rostaing L, Cantarovich D, Mourad G, et al: Corticosteroidfree immunosuppression with tacrolimus, mycophenolate mofetil, and daclizumab induction in renal transplantation. Transplantation 79:807, 2005

7. Vitko S, Klinger M, Salmela K, et al: Two corticosteroid-free regimens-tacrolimus monotherapy after basiliximab administration and tacrolimus/mycophenolate mofetil-in comparison with a standard triple regimen in renal transplantation: results of the Atlas study. Transplantation 80:1734, 2005

8. Woodle ES, Fujisawa, Steroid Withdrawal Study Group: A prospective, randomized, multicenter, double-blind study of early corticosteroid cessation versus long-term maintenance of corticosteroid therapy with tacrolimus and mycophenolate mofetil in primary renal transplant recipients: one year report. Transplant Proc 37:804, 2005

9. Kandaswamy R, Melancon J, Dunn T, et al: A prospective randomized trial of steroid-free maintenance regimens in kidney transplant recipients—an interim analysis. Am J Transplant 5:1529, 2005

10. Sinclair NR; Canadian Multicenter Transplant Study Group: Low dose steroid therapy in cyclosporine-treated renal transplant recipients with well-functioning grafts. CMAJ 147:645, 1992 